\title{
A review of hydrofluoric acid burn management
}

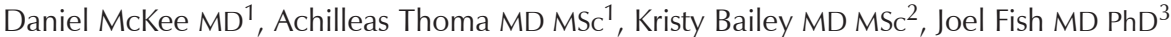

D McKee, A Thoma, K Bailey, J Fish. A review of hydrofluoric acid burn management. Plast Surg 2014;22(2):95-98.

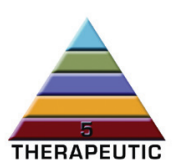

Hydrofluoric acid (HF) causes a unique chemical burn. Much of the current treatment knowledge of HF burns is derived from case reports, small case series, animal studies and anecdotal evidence. The management can be challenging because clinical presentation and severity of these burns vary widely. Plastic surgeons managing burn patients must have a basic understanding of the pathophysiology, the range of severity in presentation and the current treatment options available for HF burns.

The present article reviews the current understanding of the pathophysiology and systemic effects associated with severe HF burns. Furthermore, it distinguishes between minor and life-threatening HF burns and describes several of the basic techniques that are available to treat patients with HF burns.

Key Words: Acid; Hydrofluoric; Review

\section{Une analyse de la prise en charge des brûlures à l'acide fluorhydrique}

L'acide fluorhydrique (AF) provoque une brûlure chimique bien particulière. La plus grande partie des connaissances actuelles sur les brûlures à l'AF sont dérivées de rapports de cas, de petites séries de cas, d'études sur des animaux et d'observations isolées. La prise en charge peut être difficile, car la présentation clinique et la gravité des brûlures sont très variées. Les plasticiens qui traitent les patients brûlés doivent posséder des connaissances de base sur la physiopathologie, les divers degrés de gravité de présentation et les possibilités thérapeutiques offertes pour soigner les brûlures à l'AF.

Le présent article contient une analyse des connaissances sur la physiopathologie et les effets systémiques des graves brûlures à l'AF. Il fait également la distinction entre les brûlures à l'AF mineures et celles au potentiel fatal et contient une description de quelques-unes des techniques de base offertes pour traiter les patients ayant des brûlures à l'AF.
$\mathrm{H}$ ydrofluoric acid (HF) is an inorganic acid commonly used in many domestic and industrial settings. HF was first used for etching glass due to its corrosive properties, but currently is also widely used in cleaning agents, rust removers, in the semiconductor industry, and for manufacturing fertilizer, pesticides and some plastics.

Technically, HF is a weak acid when compared with other hydrogen halide acids, meaning the molecule does not strongly dissociate into hydrogen and fluorine ions when dissolved in water. In fact, after being dissolved in water, HF becomes much less dangerous and less acidic at low concentration $(\leq 20 \%)(1,2)$. However, at high concentrations $(>20 \%)$, small quantities of HF can cause life-threatening burns, and if the diagnosis is missed or the treatment delayed, the consequences may be devastating for the patient. The present narrative review aims to inform the reader on current management standards for these unique chemical burns.

\section{MECHANISM OF INJURY}

HF causes tissue destruction by two primary mechanisms (3):

1. At concentrations $>50 \%$, HF acidity increases dramatically and it then behaves like a strong acid. The hydrogen ion causes a corrosive burn similar to other acid burns - this damage occurs immediately and results in visible tissue destruction. However, for low-concentration HF burns - which represent the large majority of HF burns immediate corrosive destruction does not occur in any significant manner and there may be no immediate pain or tissue destruction.

2. The second, more significant mechanism of tissue destruction is caused by fluoride ions. Liquefaction necrosis of deeper tissues is unique to HF because the acid is highly lipophilic and readily penetrates deep into tissue (Figure 1). The molecule then wreaks havoc as it releases its acidic hydrogen ion and fluoride ion in the presence of cations such as calcium and magnesium. This oftendelayed reaction is responsible for the 'pain out of proportion' to physical examination findings, a result believed to be related to the local hyperkalemia effect secondary to calcium binding. Cell membrane permeability to potassium is increased by local calcium depletion; in addition, fluoride ions are believed to directly inhibit $\mathrm{Na}^{+} \mathrm{K}^{+}$pumps. Both result in local hyperkalemia, neuronal depolarization and intense pain.

Pain out of proportion to physical examination is a hallmark finding in HF burns. Clinically, the morbidity of the burn is directly proportional to the concentration of HF, the duration of exposure, and the immediacy and adequacy of first aid measures (eg, copious irrigation). In the industrial setting, concentrations can reach levels $>20 \%$; however, the majority of patients are burned at $1 \%$ to $3 \%$ concentration, more commonly present in cleaning solutions and solvents (4-6). It is useful to categorize exposures based on the concentration of acid. Higher concentrations of acid results in more immediate pain and visible burn, followed by the development of grey areas, necrosis or ulceration, and possibly tenosynovitis and osteolysis, which can present as late manifestations. Lower concentrations $(<20 \%)$ could result in delayed symptoms up to $24 \mathrm{~h}$ postexposure and, if left untreated, could progress through the same sequence as the highconcentration burns.

\section{SYSTEMIC TOXICITY}

For all cutaneous HF burns, the clinician must include an assessment of any systemic effects. Late clinical manifestations of systemic toxicity include nausea, vomiting, abdominal pain, convulsions, hypotension, cardiac arrhythmias and cardiac failure. These findings are often not present in the majority of cases with low-concentration exposure. Patients are usually asymptomatic beyond the pain in the area of cutaneous exposure.

Kirkpatrick and Burd (2), and Upfal and Doyle (3) have both provided extensive reviews of HF burns in the literature. Both describe the patient populations at risk for systemic toxicity as being any burns with $>50 \%$ concentration, exposure of $>5 \%$ total body surface area (TBSA) with any HF concentration, and inhalation or ingestion of HF $(7,8)$. However, these guidelines have had rare exceptions

${ }^{1}$ Division of Plastic and Reconstructive Surgery, Department of Surgery, McMaster University, Hamilton; ${ }^{2}$ Department of Dermatology, University of Toronto; ${ }^{3}$ Division of Plastic and Reconstructive Surgery, The Hospital for Sick Children, University of Toronto, Toronto, Ontario 


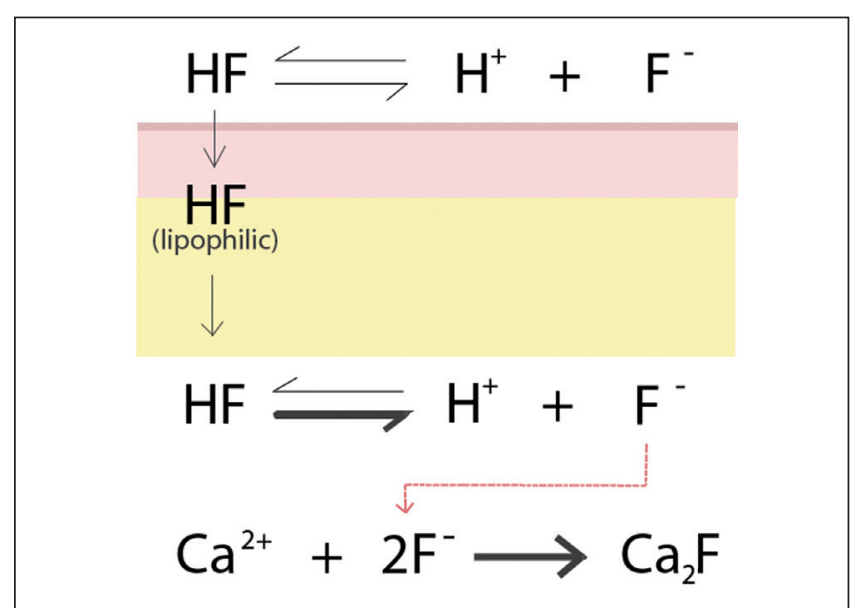

Figure 1) Hydrofluoric acid burn. Mechanism of skin penetration and underlying soft tissue destruction via dissociation of the hydrofluoric acid molecule and formation of calcium fluoride salt

described in the literature with cases not meeting the above criteria, but systemic toxicity still developing in the setting of other contributing factors such as increased duration of acid exposure (9). While systemic toxicity occurs commonly with inhalation or ingestion of HF, systemic toxicity does not manifest in minor cutaneous injuries involving low concentrations of $\operatorname{HF}(10,11)$.

The systemic effects are primarily related to electrolyte disturbances - mainly hypocalcemia - but also hypomagnesemia, acidosis, fluorosis and hyperkalemia, which can lead to disturbances of renal, hepatic and cardiac function (12,13). Fluoride ions bind calcium and magnesium, and this process may occur at a rate exceeding the body's ability to mobilize calcium and magnesium in the serum. In the majority of cases, clinical evidence of hypocalcemia is absent; therefore, high-risk patients must be evaluated by electrocardiography for prolonged QT interval and arrhythmias and placed on cardiac monitoring (Figure 2) (14). In addition to causing hypocalcemia, fluoride ions are believed to be directly toxic to myocardial cells by inhibiting adenylate cyclase. For severe HF burns, serum electrolyte levels should be obtained urgently and then frequently monitored as dictated by the degree of clinical exposure and systemic involvement. Guidelines advocate calcium replacement in high-risk groups even before the serum calcium level is determined (8). Proven hypocalcemia warrants calcium gluconate infusion parenterally and frequent serum calcium monitoring. Rapid urinary excretion and alkalization of urine have been described as effective means of fluoride ion removal. Hemodialysis has been reported to reduce both fluoride and potassium levels, and to treat persistent hypocalcemia despite calcium infusion. Some authors advise that if severe systemic toxicity develops despite intravenous calcium infusion and intensive care resuscitation, immediate surgical excision of the burn should be considered to remove the tissue source of the fluoride ions (15). This scenario is extremely rare and carries a poor prognosis. The use of urgent surgery in this setting is controversial because there are no controlled studies demonstrating any benefit over continued resuscitation.

\section{IRRIGATION}

Immediate skin surface irrigation with tap water should be initiated to remove HF from the skin and prevent rapid penetration by the extremely lipophilic acid. There is general consensus that lavage should occur immediately at the site of the accident for $15 \mathrm{~min}$ to $30 \mathrm{~min}$ before proceeding to the emergency department. "HF chemical injuries in which lavage was initiated as a first aid measure showed significantly less full-thickness injury and more than a twofold shorter hospital stay compared to those who did not receive lavage until admission to hospital" $(2,16)$. No fluid decontamination alternatives have been shown to be superior to water for first-aid irrigation (17).

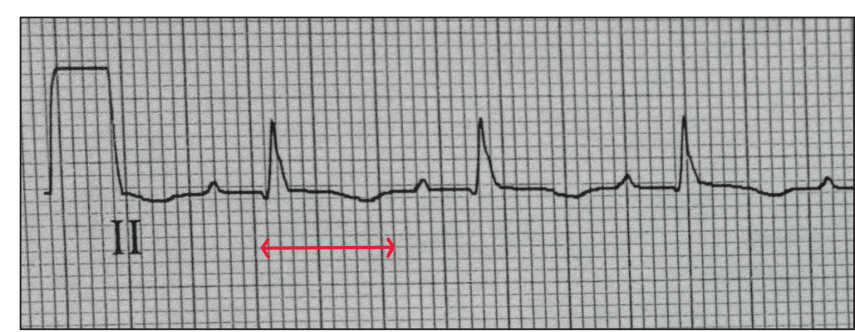

Figure 2) An electrocardiogram displaying a prolonged QT interval (arrow) secondary to hypocalcemia in a severe hydrofluoric acid burn

\section{TOPICAL GEL}

After irrigation, the next goal is to deactivate the free fluoride ions that have penetrated into deeper tissues. Historically, magnesium compounds or ammonium-based compounds (such as hyamine) were used to promote the formation of an insoluble fluoride salt, but these were either shown to be less effective compared with calcium gel or caused systemic toxicity (2). Calcium gluconate gel is standard practice to treat HF burns in most centres despite there being no controlled studies involving humans showing any benefit or to guide protocol. Calcium gluconate gel can be made in the emergency department with a water-soluble lubricant, such as K-Y Jelly (Johnson \& Johnson, USA), added to calcium gluconate solution or calcium gluconate powder ( $75 \mathrm{~mL} \mathrm{K-Y} \mathrm{Jelly} \mathrm{plus} 25 \mathrm{~mL}$ of $10 \%$ calcium gluconate or $100 \mathrm{~mL}$ of K-Y Jelly plus $2.5 \mathrm{~g}$ of calcium gluconate) $(8,18)$. The gel is applied every $30 \mathrm{~min}$ initially and massaged in, then applied every $4 \mathrm{~h}$. A clinically reliable indicator of effectiveness is pain relief $(19,20)$. The advantages of the gel are that it is easy to apply, painless and noninvasive; however, large quantities may be required and the impermeability of the skin to calcium limits its depth of penetration (21).

\section{INFILTRATION}

Animal-control studies have shown mixed results for calcium gluconate injection on HF burn healing outcomes (22). Nevertheless, emergency room physicians and surgeons have widely adopted its use for HF burns, likely because it has been shown in humans to reliably reduce pain after injection (23). The protocol first described by Dibbell et al (24) used a 27 -gauge needle to inject a $5 \%$ to $10 \%$ calcium gluconate solution into subcutaneous tissue within and beneath the burn (injecting $0.5 \mathrm{~mL} / \mathrm{cm}^{2}$ of burn surface area). As mentioned, the efficacy of infiltration is controversial in the literature, and most surgeons reserve the use of infiltration for the moderate to severe burns. Indications described in the literature include a central hard grey area with surrounding erythema (signifying a moderate to high concentration $>20 \%$ causing deep burns), and throbbing severe pain despite management with irrigation and gel. Most authors agree that infiltration is unnecessary for burns with HF concentrations $\leq 20 \%$.

Infiltration can cause increased compartment pressure and necrosis. Edelman et al (25) were first to describe this and set a limit of $0.5 \mathrm{~mL}$ per phalanx with repeated injections preferred (as opposed to one large-dose injection) (25). The amount of fluid in the pad of an adult finger is generally $<0.5 \mathrm{~mL}$ and, in our experience, there is a marked reduction in pain using this technique. Anderson and Anderson (26) proposed a palmar fasciotomy in conjunction with any injection of the finger tips because $0.5 \mathrm{~mL}$ was believed to be inadequate to neutralize sufficient fluoride ions, but this has not been widely adopted. In general, infiltration also may introduce infection and also carries the risk of hypercalcemia. Calcium salts produced from infiltration may be directly toxic to the tissue; therefore, the use of calcium chloride is generally prohibited despite its greater binding capacity. The use of a local anesthetic injection is controversial because some authors believe it either masks the pain - which is a valuable clinical indicator of treatment effectiveness - or may also further increase tissue tension. The ability to use short-term sedation, such as propofol, has allowed for repeated injections to occur and the masking effect of the local anesthetic is avoided. 


\section{INTRA-ARTERIAL INFUSION}

Intra-arterial calcium infusion is reserved for patients with severe HF burns with unrelenting pain despite aggressive calcium gel therapy (4). The technique was originally developed for high-concentration HF digital burns in which large numbers of fluoride ions need to be neutralized but available tissue space was too limited to use the infiltration method safely. Kohnlein and Achinger (27) were the first to describe intra-arterial calcium infusion in 1978 and their protocol consisted of an angiogram to guide the route of infusion to either the radial, ulnar or brachial artery. They used $50 \mathrm{~mL}$ of $4 \%$ calcium gluconate given over a $4 \mathrm{~h}$ period and repeated the cycle every $12 \mathrm{~h}$ until the patient was free from pain. However, complications occurred frequently, including artery spasm and bleeding, ulnar nerve palsy relating to the position of immobilization, median nerve palsies secondary to hematomas, carpal tunnel syndrome and hypercalcemia. Some authors have adapted this protocol and have reported encouraging results. It is traditionally used for digital burns of the hand, but has also been described for the face and lower extremities (28-30). However, some centres avoid intra-arterial infusion because cannulation of the brachial artery carries high morbidity and requires intensive care unit (ICU) monitoring. Calcium gluconate can be given intravenously with an above-elbow cuff based on Bier's method; however, this method has not been widely adopted (31-33).

\section{SURGERY}

The majority of HF burns (which are minor and low concentration) are not lethal, but simply painful, and the role of surgery is to debride blisters or to excise eschar, thereby allowing application and adequate penetration of topical agents. At the opposite extreme, there are cases in the literature in which immediate surgery was reported as crucial and lifesaving $(34,35)$. In these infrequent, severe cases, such as a $5 \%$ total body surface area burn with $>50 \%$ concentration HF in a patient who develops cardiac arrhythmias, conservative methods alone, such as infiltration, may be futile because the patient is in need of aggressive resuscitation and consideration for immediate surgical debridement. For cases that fall in the middle of the spectrum of severity, there is no consensus on indications for immediate surgical debridement, but immediate debridement is generally reserved for high-concentration burns or those that show systemic toxicity despite more conservative management. In the large majority of cases, surgery is delayed until wounds are well demarcated. Using traditional methods in gauging burn depth intraoperatively, including the pale yellow fat, pearly white dermis and patent vessels, are unreliable in chemical burns (36). It is not uncommon for debrided wounds to appear viable yet experience

\section{REFERENCES}

1. Caravati M. Acute hydrofluoric acid exposure. Am J Emerg Med $1988 ; 6: 143-50$

2. Kirkpatrick J, Burd D. Hydrofluoric acid burns: A review. Burns 1995;21:483-93.

3. Upfal M, Doyle C. Medical management of hydrofluoric acid exposure. J Occup Med 1990:726-31.

4. Stuke LE, Arnoldo BD, Hunt JL, Purdue GF. Hydrofluoric acid burns: A 15-year experience. J Burn Care Res 2008;29:893-6.

5. Hatzifotis M, Williams A, Muller M, Pegg S. Hydrofluoric acid burns. Burns 2004;30:156-9.

6. Strausburg M, Travers J, Mousdicas N. Hydrofluoric acid exposure: A case report and review on the clinical presentation and management. Dermatitis 2012;23:231-6.

7. Greco R, Hartford C, Haith L, et al. Hydrofluoric acid induced hypocalcemia. J Trauma 1988;28:1593-6.

8. Kirkpatrick J, Burd D. An algorithmic approach to the treatment of hydrofluoric acid burns. Burns 1995;21:495-99.

9. Ming-Ling W, Juo-Fang D, Ju-Sing F. Survival after hypocalcemia, hypomagnesemia, hypokalemia and cardiac arrest following mild hydrofluoric acid burn. Clin Toxicol 2010;48:953-55.

10. Burd A. Hydrofluoric acid burns: Rational treatment. J Burn Care Res 2009;30:908

11. Yolken R, Konecny P, Mccarthy P. Acute fluoride poisoning. Pediatrics 1976;58:90-3. poor graft take. Staging the surgery and incorporating multiple debridements when large areas are involved is common practice. In this case, allografts or xenografts can be used after the first debridement to enable further wound demarcation while keeping the wound closed to contamination. This method also spares the donor sites until the wound bed is certain to support a graft or flap.

\section{NAIL INJURIES}

HF readily passes through and around the nail plate causing severe damage to the delicate subungual tissues. Involvement of the nail bed poses a unique challenge in calcium gel delivery and removal of the fingernail is often required. One should not hesitate to remove the nail if severe pain is present. This requires local block or sedation and the finger pads can be injected at the same time (9).

\section{OCULAR INJURIES}

The eye tissue is highly susceptible to HF liquid or vapour (37). Similar to cutaneous burns, the most important therapy is efficient immediate lavage. Some authors describe using 1\% to $10 \%$ calcium gluconate eye drops after irrigation. A prompt ophthalmological opinion is mandatory if any concerns arise.

\section{CONCLUSION}

Much of the current treatment knowledge of HF burns is derived from case reports, small case series, animal studies and anecdotal evidence. The management of these unique chemical burns can be challenging because clinical presentation and severity of these burns vary widely. The most common presentation of an HF burn is a small area, usually to the hands, involving a low concentration of HF. Monitoring of electrolyte levels and ICU admission should not be a part of the management practice in these small HF burns caused by common workplace and household chemicals (4). However, several case reports have shown that severe HF burns can be life threatening, requiring ICU admission and urgent surgical intervention $(31,38)$. Current treatment techniques for HF burns have generally been unchanged over the past two decades in the published literature. However, through gained knowledge and experience of these unique chemical burns, clinicians are now better able to quickly distinguish between minor and lifethreatening HF burns and manage accordingly.

DISCLOSURES: The authors have no financial disclosures or conflicts of interest to declare.

12. Mclvor M. Delayed fatal hyperkalemia in a patient with acute fluoride intoxication. Ann Emerg Med 1987;16:118-20.

13. Sanz-Gallen P, Nogue S, Munne P. Hypocalcemia and hypomagnesemia due to hydrofluoric acid. Occup Med 2001;51:294-5.

14. Holstege C, Baer A, Brady W. The electrocardiographic toxidrome: The ECG presentation of hydrofluoric acid ingestion. Am J Emerg Med 2005;23:171-6.

15. Ohtani M, Nishida N, Chiba T, et al. Pathological demonstration of rapid involvement into the subcutaneous tissue in a case of fatal hydrofluoric acid burns. Forensic Sci Int 2007;167:49-52.

16. Leonard L, Scheulen J, Munster A. Chemical burns: Effect of prompt first aid. J Trauma 1982;22:420-3.

17. Hulten P, Hojer J, Ludwigs U, et al. Hexaluorine vs. standard decontamination to reduce systemic toxicity after dermal exposure to hydrofluoric acid. J Toxicol Clin Toxicol 2004:42:355-61.

18. Lukinuk C, Dasgupta R, Mahoney J. Hydrofluoric acid burns: A case report. Can J Plast Surg 1997;5:179-80.

19. Ohata U, Hara H, Suzuki H. 7 cases of hydrofluoric acid burn in which calcium gluconate was effective for relief of severe pain. Contact Dermatitis 2005;52:133-7.

20. Foster K, Jones L, Caruso F. Hydrofluoric acid burn resulting from ignition of gas from a compressed air duster. J Burn Care Rehabil 2003;24:234-7. 
21. Chick L, Borah G. Calcium carbonate gel therapy for hydrofluoric acid burns of the hand. Plast Recon Surg 1990;86:935-40.

22. Stacy S, Noordhoek L, Botens S, et al. A study to determine the efficancy of treatments for hydrofluoric acid burns. J Burn Care Rehabil 1995;16:253-7.

23. Carpenter B, Wolfort F, Tubridy S, et al. Hydrofluoric acid burns of the lower extremity. J foot Ankle Surg 1999;38:366-9.

24. Dibbell D, Iverson R, Laub D, et al. Hydrofluoric acid burns of the hand. J Bone Joint Surg 1970;52:931-6.

25. Edelman P. Hydrofluoric acid burns. Occup Med 1986;1:89-103.

26. Anderson W, Anderson J. Hydrofluoric acid burns of the hand: Mechanism of injury and treatment. J Hand Surg Am 1988;13:52-7.

27. Kohnlein H, Achinger R. A new method of treatment of hydrofluoric acid burns of the extremities. Chir Plast 1982;6:297-305.

28. Lin T, Tsai C, Lin S. Continuous intra-arterial infusion therapy in hydrofluoric acid burns. J Occup Envirn Med 2001;42:892-7.

29. Nguyen L, Mohr W, Ahrenholz D, et al. Treatment of hydrofluoric acid burns to the face by carotid artery infusion of calcium gluconate. J Burn Care Rehabil 2004;25:421-2.
30. Thomas D, Jaeger U, Sagoschen I, et al. Intar-arterial calcium gluconate treatment after hydrofluoric acid burn of the hand. Cardiovasc Intervent Radiol 2009;32:155-8.

31. Wedler V, Guggenheim M, Moron M, et al. Extensive hydrofluoric acid injuries: A serious problem. J Trauma 2005;58:852-7.

32. Henry J, Hla K. Intravenous regional calcium gluconate perfusion for hydrofluoric acid burns. J Toxicol Clin Toxicol 1992;30:203-7.

33. Gupta R. Intravenous calcium gluconate in the treatment of hydrofluoric acid burns. Ann Emerg Med 2001;37:724-4.

34. Burd A. Hydrofluoric acid - revisited. Burns 2004;30:720-2.

35. Buckingham F. Surgery: A radical approach to severe hydrofluoric acid burns. J Occup Med 1988;30:873-4.

36. Bezuhly M, Fish J. Acute burn care. Plast Reconstr Surg 2012;130:349e-58e.

37. Matsuno K. The treatment of hydrofluoric acid burns. Occup Med 1996;46:313-7.

38. Dünser MW, Ohlbauer M, Rieder J, et al. Critical care management of major hydrofluoric acid burns: A case report, review of the literature, and recommendations for therapy. Burns 2004;30:391-8. 